\title{
基于一维流动模型的高速列车隧道压力波特性
}

\author{
贾永兴 杨永刚 梅元贵 \\ (兰州交通大学机电工程学院 兰州 730070)
}

\begin{abstract}
摘要: 高速列车通过隧道过程中引起隧道内压力的剧烈波动, 会诱发车内压力波动并可能引起车体疲劳破坏等问题。而研究 此类问题的基础在于快速准确预测隧道压力波。基于一维可压缩非定常不等熵流动模型和广义黎曼变量特征线法, 对单车通 过隧道和两列车隧道内交会进行数值模拟。选取京沪高速铁路隧道为研究对象, 通过全时间区域下隧道空间中的压力传播的 过程图描述压力波的形成过程, 给出隧道内交会压力波比单车通过隧道的压力波剧烈的原因, 研究列车速度和阻塞比对车外 最大压力值和最小压力值的影响特性。结果表明, 高速列车通过京沪高铁典型长度隧道时, 其车体表面承受的最大压力波动 基本与车速的平方成正比，而其与阻塞比基本呈线性关系。
\end{abstract}

关键词: 高速列车; 隧道压力波; 一维可压缩非定常不等熵流动模型; 特征线法

中图分类号: U238; V211

\section{Characters of Pressure Wave Caused by High-speed Trains Passing Tunnels Based on 1D Non-homentropic Flow Model}

\author{
JIA Yongxing YANG Yonggang MEI Yuangui \\ (School of Mechatronic Engineering, Lanzhou Jiaotong University, Lanzhou 730070)
}

\begin{abstract}
When high-speed trains travel through tunnels, drastic pressure variations are generated. The pressure waves may cause aural discomfort and fatigue failure of the car body, which the basic problem is predicting the pressure wave rapidly. Computer programs which can predict the pressure wave caused by a single train or two crossing trains in the tunnel are introduced and validated. One-dimensional(1D), compressible, unsteady, and non-homentropic flow model and method of characteristics of generalized Riemann variables are used in these programs. Railway tunnel with typical length in Beijing-Shanghai high-speed rail line are studied. The generation of pressure wave is depicted through the spatial and temporal variations in pressure. The reason the pressure wave caused by two crossing trains is severer than that by the single train is qualitatively analyzed. And the effect of train speed and blockage ratio on the maximum and minimum external pressure excursion is studied. The result shows that the maximum pressure excursion is approximately proportional to the square of train speed and linear related to the blockage ratio when high-speed trains passing through or crossing in the tunnel of typical length in Beijing-Shanghai high-speed rail line.
\end{abstract}

Key words: high-speed trains; pressure wave in railway tunnels; one-dimensional unsteady compressible non-homentropic flow model; method of characteristics

\section{0 前言}

随着高速列车运营速度的不断提高, 高速列车 空气动力问题变得越来越重要。单列车通过隧道或 两列车在隧道内交会时, 空气流动受到隧道壁面的 限制, 使得高速列车隧道空气动力学问题比明线时 更加剧烈。当高速列车通过隧道时, 隧道内产生的

* 国家重点基础研究发展计划(973 计划, 2011CB711101)和国家自然科学 基金(51065013)资助项目。20131225 收到初稿, 20140707 收到修改稿
剧烈压力波动传播到车内后会引起旅客的耳感不适 问题 ${ }^{[1-2]}$ 。所以, 在中国高速铁路线上, 为保证旅客 的舒适性, 采用了安装气密装置的车辆。同时, 压 力波作用在列车车体上时, 会引起了交变的气动载 荷。这就需要在车辆设计初期估算出压力波动的大 小。因此, 有必要研究列车通过隧道产生的流动和 压力波的特性。

通常情况, 隧道长度远大于隧道当量水力直 径。此时, 列车通过隧道引起的空气流动可简化为 一维可压缩非定常流动。这也为快速准确分析压力 
波特性提供了理论基础。自 20 世纪 60 年代开始, 国内外学者大致使用如下四种一维模型 ${ }^{[2-3]}$ 进行隧 道压力波的研究, 具体可分类如下: (1) 定密度有限 声速非定常流动模型 ${ }^{[4-5]}$; (2) 可压缩非定常等熵流 动模型 ${ }^{[6]}$; (3) 考虑摩擦的可压缩非定常等熵流动模 型 ${ }^{[7-8]}$; (4) 可压缩非定常不等熵流动模型 ${ }^{[9-10]}$ 。

模型(1)将列车端部的流动简化为不可压缩流 动, 主要针对列车运营速度远小于当地声速的情况。 当列车速度大于 $350 \mathrm{~km} / \mathrm{h}$ 时, 这种假设引起的误差 较大 ${ }^{[10]}$ 。德国学者早期主要使用忽略了列车和隧道 壁面与空气之间的摩擦效应的模型(2), 该模型在计 算较长隧道时误差较大。模型(3)假设空气流动为等 熵过程, 但在动量方程中考虑了隧道内空气与隧道 壁面、列车壁面间的摩擦力。该模型能够比较准确 地模拟压力波动和平均流速, 容易应用在复杂隧道 中的多列车的情形, 但由于没有考虑热交换, 在模 拟更高速度列车通过长隧道情形隧道气温变化的误 差较大 ${ }^{[11]}$, 也不能应用于车辆内部与隧道之间质量 交换、隧道壁面开孔等情形。

模型(4)严格根据一维可压缩非定常不等熵流 动理论建立隧道内空气流动模型。该模型考虑了空 气与隧道壁面、列车壁面之间的摩擦与传热等不可 逆因素, 以及隧道内环状空间与车内空间因压差而 导致的质量交换。WOODS 等 ${ }^{[12]}$ 基于该模型，采用 广义黎曼变量特征线法, 发展了单列车通过隧道的 流动的数值计算方法, 但没有应用到多列车运行和 复杂隧道结构中。此后, STEINRÜCK 等 ${ }^{[13]}$ 假设列 车端部交会过程中的流动为不可压缩流动, 将该模 型扩展到研究两列车在隧道内交会时引起的压力波 动; 上述流动模型的数学方程组均为一阶拟线性双 曲型方程组, 而特征线法是求解此类方程的主要方 法。特征线法准确快速, 且使用一般的计算机即可 完成计算研究工作。我国自 20 世纪 90 年代开始系 统研究高速列车隧道空气动力学问题。梅元贵等 $[2,14]$ 采用广义黎曼变量特征线法发展了隧道内单车压力 波和会车压力波的计算方法和计算程序。

随着计算机水平的不断提升, 越来越多的国内 外学者开始使用商用软件对高速列车隧道空气动力 学问题进行二维或三维数值模拟研究。采用三维数 值模拟研究高速列车通过隧道产生的空气流动问题 时要求综合考虑流动不稳定性、可压缩性、湍流流 动等各方面的要求。由于三维数值模拟计算量过大 且其研究问题可用二维模型准确描述, 陶泽平等 ${ }^{[15]}$ 采用计算流体力学方法的动态分层模型研究了列车 隧道交会时最不利长度问题。王一伟等 ${ }^{[16]}$ 为避免三 维验证工作的巨大计算量, 建立了简化的二维隧道
会车计算方法, 研究了隧道长度对高速列车隧道交 会压力波的影响。

KRAJNOVIC 等 ${ }^{[17]}$ 基于 STAR-CD 和超级计算 机进行了简化 ICE2 高速列车在隧道内车外流动分 布特性的大涡模拟, 并预测在未来 50 年内仍不能采 用直接数值模拟方法模拟列车通过隧道时诱发空气 流动的精细特征。可见计算机水平的限制仍是限制 商用计算流体力学(Computational fluid dynamics, CFD)软件推广的主要瓶颈 ${ }^{[18]}$ 。目前使用三维数值模 拟方法的研究热点仍集中于局部特征, 如高速列车 驶入隧道过程 ${ }^{[19]}$ 、隧道端口缓冲结构对隧道压力波 的影响 ${ }^{[20]}$ 以及压力波对高速铁路局部结构 ${ }^{[21-23]}$ 的 影响等。李人宪等 ${ }^{[24]}$ 使用计算流体力学三维数值分 析方法研究了隧道内会车时运行列车上的变化规 律，得到了最大隧道内可能的最大负压峰值计算式 及其与会车速度的关系式; 李人宪等 ${ }^{[22]}$ 采用同样的 方法对明线和隧道内会车时压力波动对侧窗的影响 进行了研究。近年来, 相关研究机构的科研人员对 隧道压力波的影响进行了更加深入细致的研究。施 成华等 ${ }^{[21]}$ 研究了隧道压力波负压作用对水沟盖板 稳定性的影响, 认为 $350 \mathrm{~km} / \mathrm{h}$ 会车时, 会引起水沟 盖板的微量位移和响动, 应增大手孔尺寸以避免噪 声和安全性问题。费瑞振等 ${ }^{[23]}$ 对高速铁路隧道压力 波空气动力效应对人员安全性进行了研究, 并给出 了安全速度限值。

综述所述，虽然超级计算机及并行计算技术在 一定程度上拓展了三维数值模拟方法研究高速列车 隧道空气动力学问题的范围, 但其仍无法满足工程 设计初期快速多工况预测的要求。本文在介绍所采 用一维可压缩非定常不等熵流动模型基础上, 以京 沪高速铁路隧道为背景, 分析了单列车通过隧道和 两列车隧道内交会引起压力波的特点。研究了车外 最大压力值和最小压力值随列车速度和阻塞比的变 化特性。

\section{1 一维不等熵流动模型}

\section{1 控制方程及数值模拟方法}

高速列车通过隧道产生的空气流动实际上伴 随有分离流动和传热的三维、不稳定、可压缩的湍 流流动。当隧道长度远大于隧道当量水力直径时, 除列车端部和隧道洞口区域外，隧道内的大部分流 动可简化为一维可压缩不稳定流动。本文研究的隧道 为等截面且不设置通风坚井等辅助结构的水平放置 的简单隧道; 考虑空气与隧道壁面、列车表面之间的 摩擦和传热; 列车车辆绝对密封(车厢内外的空气质 
量交换); 假设隧道内的空气是理想气体。因此, 一 维可压缩非定常不等熵流动模型的控制方程 ${ }^{[2]}$ 如下。 连续性方程

$$
\frac{\partial \rho}{\partial t}+u \frac{\partial \rho}{\partial x}+\rho \frac{\partial u}{\partial x}=0
$$

动量方程

$$
\frac{\partial u}{\partial t}+u \frac{\partial u}{\partial x}+\frac{1}{\rho} \frac{\partial p}{\partial x}+G=0
$$

能量方程

$$
\begin{gathered}
\left(\frac{\partial p}{\partial t}+u \frac{\partial p}{\partial x}\right)-a^{2}\left(\frac{\partial \rho}{\partial t}+u \frac{\partial \rho}{\partial x}\right)= \\
(q-w+u G) \rho(\kappa-1)
\end{gathered}
$$

式中 $u, a$ 一流速度和声速;

$\rho, p, \kappa$ 一一空气密度, 压力和比热容比;

$g$ 一重力加速度;

$w$ 一一列车动壁面对空气所做的功;

$G$ 一摩擦力项;

$Q$ - 空气与壁面传热项;

$t$ 一一时间。

对于不含列车的流动空间

$$
\begin{gathered}
G=\frac{S_{\mathrm{TU}}}{2 F_{\mathrm{TU}}} f_{\mathrm{TU}} u|u| \\
q=\frac{S_{\mathrm{TU}}}{2 F_{\mathrm{TU}}} \frac{\kappa R}{\kappa-1} f_{\mathrm{TU}}|u|\left(T_{\mathrm{TU}}-T\right) \\
w=0
\end{gathered}
$$

对于含有单列车的环状空间

$$
\begin{gathered}
G=\frac{1}{2 F_{\mathrm{ANTR} 1}} \mu_{\mathrm{TU}} u|u| S_{\mathrm{TU}}+ \\
\frac{1}{2 F_{\mathrm{ANTR} 1}} \mu_{\mathrm{TR}}(u-v)|u-v| S_{\mathrm{TR}} \\
q=\frac{k R}{2(\kappa-1) F_{\mathrm{ANTR} 1}} \mu_{\mathrm{TU}}|u| S_{\mathrm{TU}}\left(T_{\mathrm{TU}}-T\right)+ \\
\frac{k R}{2(\kappa-1) F_{\mathrm{ANTR} 1}} \mu_{\mathrm{TR}}|u-v| S_{\mathrm{TR}}\left(T_{\mathrm{TR}}-T\right) \\
w=\frac{S_{\mathrm{TR}}}{2 F_{\mathrm{ANTR} 1}} \mu_{\mathrm{TR}} v(u-v)|u-v|
\end{gathered}
$$

对于含有两列车的环状空间

$$
\begin{gathered}
G=\frac{1}{2 F_{\mathrm{ANTR} 2}} \mu_{\mathrm{TU}} u|u| S_{\mathrm{TU}}+ \\
\frac{1}{2 F_{\mathrm{ANTR} 2}} \mu_{\mathrm{TR} 1}\left(u-v_{1}\right)\left|u-v_{1}\right| S_{\mathrm{TR} 1}+ \\
\frac{1}{2 F_{\mathrm{ANTR} 2}} \mu_{\mathrm{TR} 2}\left(u-v_{2}\right)\left|u-v_{2}\right| S_{\mathrm{TR} 2}
\end{gathered}
$$

$$
\begin{gathered}
q=\frac{1}{2 F_{\mathrm{ANTR} 2}} \frac{\kappa R}{\kappa-1} \mu_{\mathrm{TU}} S_{\mathrm{TU}}|u|\left(T_{\mathrm{TU}}-T\right)+ \\
\frac{1}{2 F_{\mathrm{ANTR} 2}} \frac{\kappa R}{\kappa-1} \mu_{\mathrm{TR} 1} S_{\mathrm{TR} 1}\left|u-v_{1}\right|\left(T_{\mathrm{TR} 1}-T\right)+ \\
\frac{1}{2 F_{\mathrm{ANTR} 2}} \frac{\kappa R}{\kappa-1} \mu_{\mathrm{TR} 2} S_{\mathrm{TR} 2}\left|u-v_{2}\right|\left(T_{\mathrm{TR} 2}-T\right) \\
w=\frac{S_{\mathrm{TR} 1}}{2 F_{\mathrm{ANTR} 2}} \mu_{\mathrm{TR} 1} V_{1}\left(u-v_{1}\right)\left|u-v_{1}\right|+ \\
\frac{S_{\mathrm{TR} 2}}{2 F_{\mathrm{ANTR} 2}} \mu_{\mathrm{TR} 2} V_{2}\left(u-v_{2}\right)\left|u-v_{2}\right|
\end{gathered}
$$

式中 $\mu_{\mathrm{TU}}$ 一隧道壁面摩擦因数;

$\mu_{\mathrm{TR}}$ 一列车壁面摩擦因数;

$R, v$ 一理想气体常数和列车速度;

$T, T_{\mathrm{TU}}$ 一隧道内空气及隧道壁面的温度;

$S_{\mathrm{TU}}, S_{\mathrm{TR}}$ 一隧道和列车横截面的周长;

$F_{\mathrm{TU}}$ 一无车隧道空间横截面积;

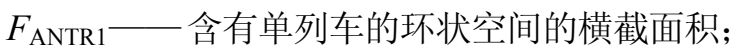

$F_{\mathrm{ANTR} 1}=F_{\mathrm{TU}}-F_{\mathrm{TR}}$;

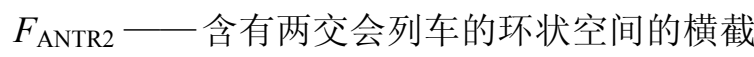
面积;

$F_{\mathrm{ANTR} 2}=F_{\mathrm{TU}}-F_{\mathrm{TR} 1}-F_{\mathrm{TR} 2} ;$

下标 1,2 - 列车 1 和列车 2 。

采用一维可压缩流动模型模拟高速列车通过 隧道引起的空气流动时, 隧道端口及列车端部边界 条件可用一维准稳定不等熵流动模型描述, 与模型 (4)对应，具体见文献[2]。对于隧道内两列车交会的 情形, 除需要建立单车通过隧道时的边界条件外, 还需要构造两列车端部两两分别交错期间端部的边 界条件。由于交错时间非常短, 可假设此时列车端 部周围的流动为不可压缩流动, 这样就可将模型(4) 推广到模拟两列车交会引起的空气流动, 具体见文 献 $[2,13]$ 。

方程式(1) (3)组成的一阶拟线性双曲型偏微 分方程组通常可采用特征线法求解。采用特征线方 法求解时, 首先需要将上述方程组转换为有量纲的 气流速度 $u$, 压力 $p$ 和密度 $\rho$ 表示的特征方程。然 后将该特征方程转换为由量纲一广义黎曼变量 $\lambda$ 和 $\beta$ 以及表示空气质点熵值的量 $A_{A}$ 表示的方程。此处 不再赘述 ${ }^{[2]}$ 。

\section{2 验证}

本文采用日本国铁技术研究所的缩尺模型试 验 ${ }^{[25]}$ 的测试结果和意大利佛罗伦萨至罗马间高速 铁路线上的 Terranuova le ville 隧道实车试验 ${ }^{[26]}$ 的测 试结果, 分别与本文程序计算得到的单车列车通过 隧道和隧道内交会时压力波动数据进行对比。并进 一步验证数学模型和本文计算方法的正确性。计算 
程序的输入数据具体见文献[25-26]。图 1 比较了本 文计算程序计算值与文献试验结果, 表明两者吻合 良好。对于单车通过隧道的情形, 给出了距隧道模 型进口端 $2 \mathrm{~m}$ 测点的隧道内静压波动值, 列车模型 速度为 $413 \mathrm{~km} / \mathrm{h}$ 。对于隧道内交会的情形, 给出了 列车第 2 节车厢外的压力波值, 两列车的速度相等, 速度为 $250.6 \mathrm{~km} / \mathrm{h}$, 两列车进入隧道的相错时间约 为 $25 \mathrm{~s}$ 。

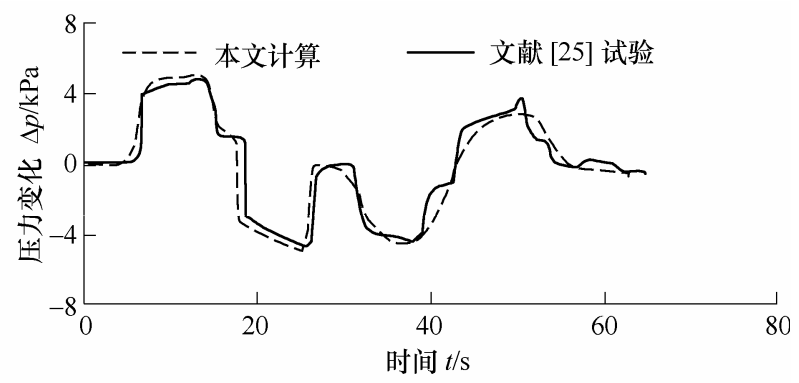

(a) 距隧道入口 $2 \mathrm{~m}$ 处

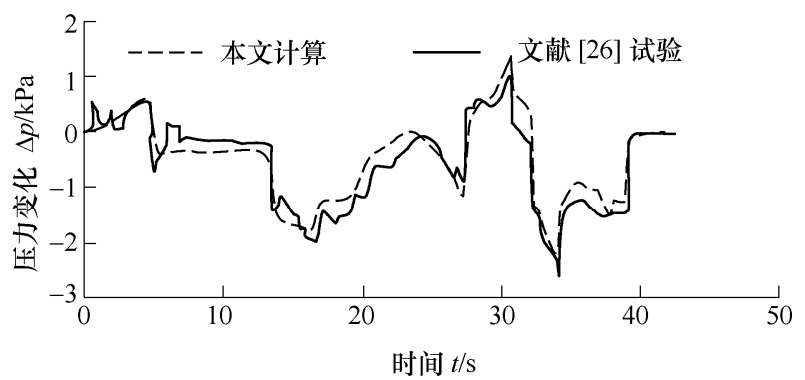

(b) 第二节列车车外中央处

图 1 本文数值计算方法验证

\section{2 隧道压力波特征}

高速列车通过隧道引起了隧道内复杂的压力 场。列车车头驶入或驶出隧道端口会产生压缩波。 类似地, 列车车尾驶入或驶出隧道将产生膨胀波。 这些波在隧道内以声速沿隧道传播, 在隧道内形成 了复杂的波干涉和叠加。在其传播至隧道洞口和列 车端部时, 会发生反射和折射。如压缩波传播至隧 道洞口会向洞内反射回膨胀波。膨胀波在洞口会向 洞内反射回压缩波。这些波在洞内相遇叠加会产生 剧烈的压力波动, 形成压力波。对于同类性质的波, 波叠加强化波的变化幅值, 在极短时间内引起了剧 烈的压力梯度。此外, 列车通过隧道内测点时, 也 会引起测点压力剧烈波动。此外, 两列车在隧道交 会, 也必引起更加剧烈的压力波动。本节选择京沪 高铁上典型长度的隧道研究隧道压力波的变化特 征, 高速列车和隧道的几何参数见表 1 。图 2 中两 列车的相错时间为 $0 \mathrm{~s}$, 也即两列车相向同时驶入 隧道。
图 2 分别表示了单车通过和两列车在 $2000 \mathrm{~m}$ 隧道内交会时, 隧道内距隧道入口 $1000 \mathrm{~m}$ 和第 8 节车外中央处的压力波动时间历程曲线。由图 2 可 见, 高速列车通过隧道会产生剧烈复杂的压力波动。 而两列车在隧道内交会产生的压力波比单车通过隧 道产生的压力波要剧烈得多。对于距离隧道入口 $1000 \mathrm{~m}$ 处测点, 单车通过隧道全过程中引起的压 力波动最大值为 $3.172 \mathrm{kPa}$ ，最小值为 $-4.087 \mathrm{kPa}$ 。 而两列车在隧道内交会时则分别为 $6.941 \mathrm{kPa}$ 和 $-8.409 \mathrm{kPa}$ 。交会时的最大压力波动值、最小压力 波动值分别是单车时的 2.19 倍和 2.06 倍。对于列车 第 8 节车外中央处测点, 交会时的最大压力波动值 为 $1.142 \mathrm{kPa}$, 单车的最大压力波动值为 $0.639 \mathrm{kPa}$ 。 交会时的最小压力波动值为 $-8.398 \mathrm{kPa}$, 单车的最 小压力波动值为 $-3.843 \mathrm{kPa}$ 。交会时的最大压力值、 最小压力值分别是单车时的 1.79 倍和 2.19 倍。

表 1 计算使用的典型的隧道和列车参数

\begin{tabular}{lcc}
\hline & 参数 & \multicolumn{2}{c}{ 数值 } \\
\cline { 2 - 4 } & 隧道 & 列车 \\
\hline 横截面积 $/ \mathrm{m}^{2}$ & 100 & 11.2 \\
横截面湿 $/$ 周 $/ \mathrm{m}$ & 35.98 & 12.4 \\
壁面摩擦因数 & 0.0050 & 0.0028 \\
长度 $/ \mathrm{m}$ & 2000 & 400 \\
隧道入口入流损失系数 & 0.5 & - \\
车头压力损失系数 & - & 0.0039 \\
车尾压力损失系数 & - & 0.0146 \\
车速 $/(\mathrm{km} / \mathrm{h})$ & - & 380 \\
阻塞比 $($ 列车横截面积与隧道横截面面积之比 $)$ & 0.112 & - \\
\hline
\end{tabular}

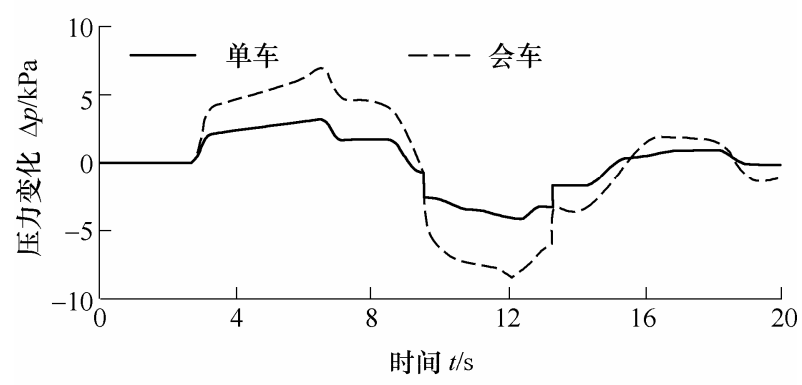

(a) 距离隧道入口 $1000 \mathrm{~m}$ 处

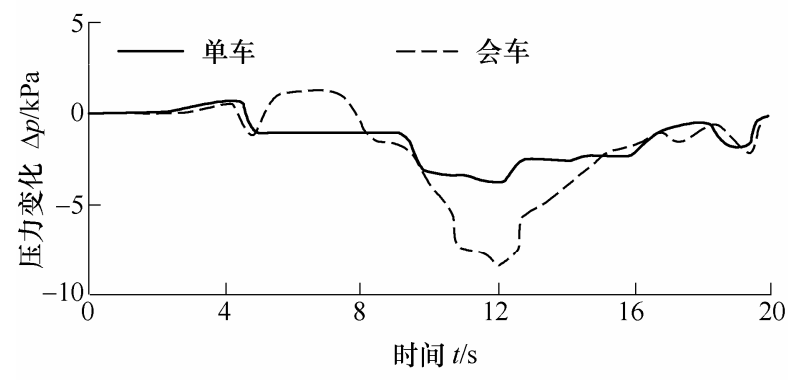

(b) 第 8 节车外中央处

图 2 单车隧道压力波和会车隧道压力波比较 
图 3 所示为两列车进入隧道的相错时间对隧道 内距隧道入口 $1000 \mathrm{~m}$ 处和和列车第 8 节车外中央 处的压力波动的影响。图 3 中列车进入隧道的相错 时间分别是 $0 \mathrm{~s} 、 6.31 \mathrm{~s}$ 和 $13.26 \mathrm{~s}$ 。相错时间 $0 \mathrm{~s}$ 意 味着两列车同时进入隧道, 在隧道中央处开始交会; 相错时间 $6.31 \mathrm{~s}$ 意味着两列车在隧道长度的 $1 / 3$ 处 开始交会; 相错时间 $13.26 \mathrm{~s}$ 意味着两列车在隧道洞 口内 $300 \mathrm{~m}$ 开始交会, 交会时晚进入隧道的列车部 分车身在隧道外。由图 3 可见相错时间对压力波的 影响。两列车在隧道内不同位置交会产生的压力时 间历程曲线有显著区别, 其最大压力波动值和最大 压力波动值也不同。从本文的研究算例发现, 两列 车同时进入隧道, 在隧道中央处开始交会的最大压 力波动值和最小压力波动值比其他两种交会位置的 压力波峰值都要大。隧道中央处开始交会的最大压 力波动值分别是隧道长度 $1 / 3$ 处交会和洞口 $300 \mathrm{~m}$ 处交会的 2.21 倍和 1.71 倍。而中央会车的最小压力 波动值分别是隧道长度 $1 / 3$ 处交会和洞口 $300 \mathrm{~m}$ 处 交会的 1.99 倍和 2.06 倍。因此本文后续内容以最恶 劣工况一一央位置会车为研究对象。

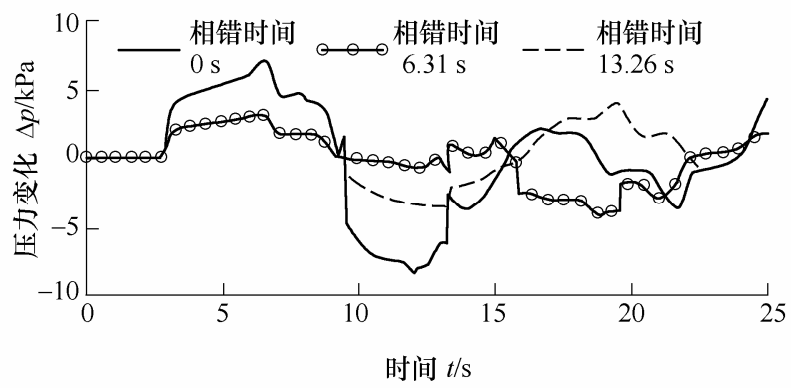

(a) 距离隧道入口 $1000 \mathrm{~m}$ 处

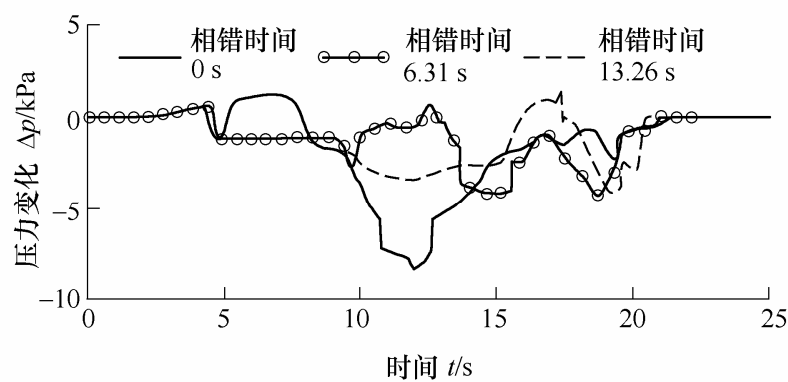

(b) 第 8 节车外中央处

图 3 两列车进入隧道相错时间不同时压力波时间历程曲线

图 4 表示了在全时间区域下在隧道空间中的压 力波传播过程。图 4 中清楚显示了压缩波和膨胀波 产生、传播和叠加过程。当列车头部进入隧道和驶 出隧道过程中, 均在隧道内产生了压缩波, 不同的 是车头进入隧道产生的压缩波强度大于驶出隧道产 生的压缩波强度。车尾进入隧道和驶出隧道均会产 生膨胀波，其两者的强度也不相同。从图 4 中还可 发现: 隧道内交会情形下的压力波比单车通过隧道
过程的压力波要剧烈的原因。交会情形下的最大压 力波动值比单车通过隧道对应的压力峰值要大 2.19 倍, 是因为两列车分别进入隧道分别产生的压缩波 在隧道内叠加所致。在列车交会过程中，负压波动 叠加产生了更加剧烈的压力波动。如此强烈的压力 波动在单车通过隧道情形下是没有的，由此使得隧 道内测点和车外测点的最小压力波动值分别为单车 通过隧道情形下的 2.06 倍和 2.19 倍。

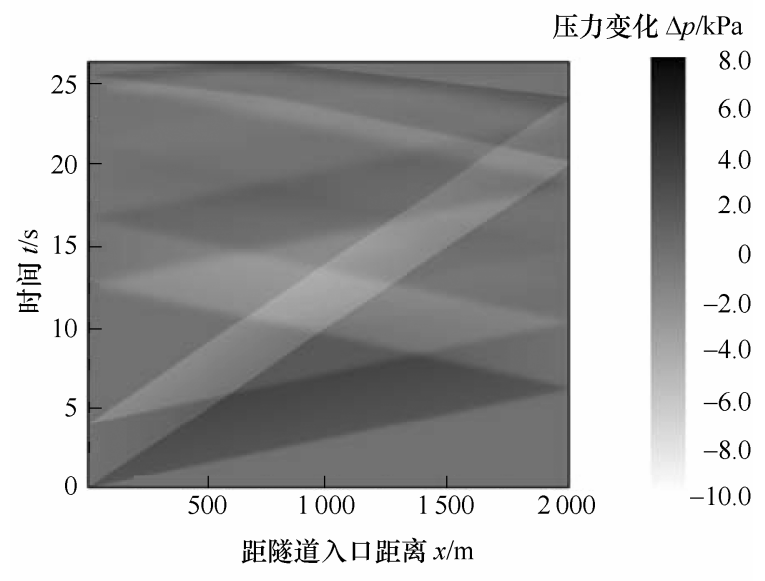

(a) 单车通过隧道引起的压力场

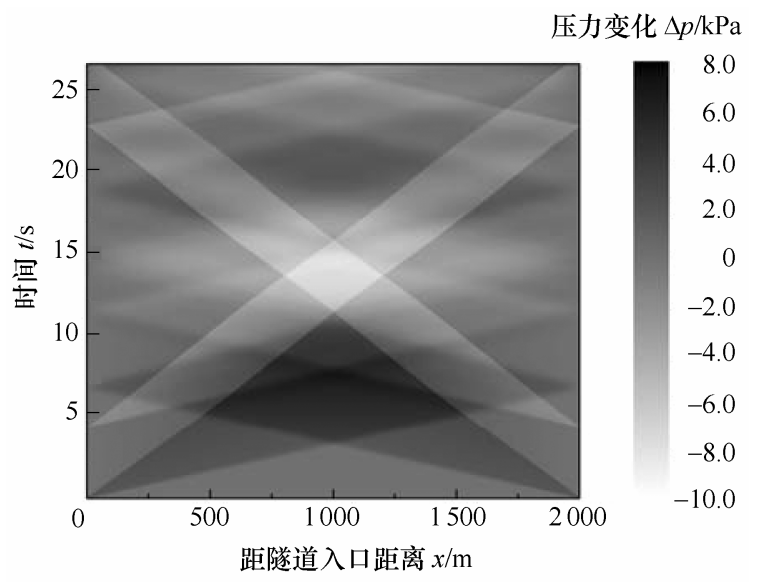

(b) 两列车隧道内交会引起的压力场

图 4 隧道空间和时间全区域下压力波的变化过程

\section{3 参数研究}

隧道和列车两方面参数影响高速列车隧道压 力波变化特性, 具体包括列车速度, 阻塞比(列车横 截面积与隧道横截面面积之比)、隧道内运行的列车 对数、隧道的辅助结构(如通风坚井)、隧道长度与列 车长度、列车和隧道表面的粗粘度、车头与车尾的压 力损失系数等。在这些影响因素中, 前 5 个因素是重 要的影响参数。无论对于单车压力波还是会车压力 波, 列车速度和阻塞比都是最重要的影响参数 ${ }^{[27]}$ 。

京沪高速铁路线有 22 座隧道, 均为单孔复线 平直的简单结构隧道, 隧道内没有辅助结构。最长 
隧道长度为 $2812 \mathrm{~m}$, 最短隧道长度 $153 \mathrm{~m}$ 。本节将 对单列高速列车通过隧道及两列车在隧道内交会的 情形进行研究, 并对车速和阻塞比对压力波的影响 进行深入探讨。

本节数值计算典型的计算输入参数见表 1 , 假 定在阻塞比 $R_{\mathrm{B}}$ 为 0.112 时, 车头压力损失系数 $\zeta_{N B}$ 和 列车表面摩擦因数 $\mu_{\mathrm{TRB}}$ 分别为 0.0039 和 0.0028 。 如果阻塞比变化, 这两个试验系数将要改变, 其近 似估算方法如下 ${ }^{[28]}$

$$
\begin{gathered}
\zeta_{\mathrm{NA}}=\left(\frac{R_{\mathrm{A}}}{R_{\mathrm{B}}}\right)^{2} \zeta_{\mathrm{NB}} \\
f_{\mathrm{TRA}}=\frac{\mu_{\mathrm{TRB}}\left(1+2.21 R_{\mathrm{A}}\right)}{1+2.21 R_{\mathrm{B}}}
\end{gathered}
$$

\section{1 列车速度的影响特性}

本文研究的列车长度为 $400 \mathrm{~m}$, 由 16 节车辆编 组而成。这里, 仅给出第 8 节车厢外纵向中央横截 面处测点的压力变化。图 5 和图 6 分别表示了列车 速度对车外压力波动峰值的影响特性。由图 5、6 可见, 随列车速度的提高, 车外的最大压力波动值 增大, 而最小压力波动值减少, 其压力变化量近似 与列车速度平方成正比。若采用 $\Delta p- \pm v^{n}$ 表示上述 关系，通过拟合，可得到 $n$ 的取值范围。在图 5、6 中所研究的速度范围内, 单列高速列车通过 $2000 \mathrm{~m}$ 隧道时, 对于最大压力波动值 $n=1.913 \sim 2.034$, 对 于最小压力波动值 $n=1.896 \sim 2.140$; 而两列车在 $2000 \mathrm{~m}$ 隧道内交会时, 对于最大压力波动值 $n=1.730 \sim 2.630$, 对于最小压力波动值 $n=1.969 \sim$ 1.987 。

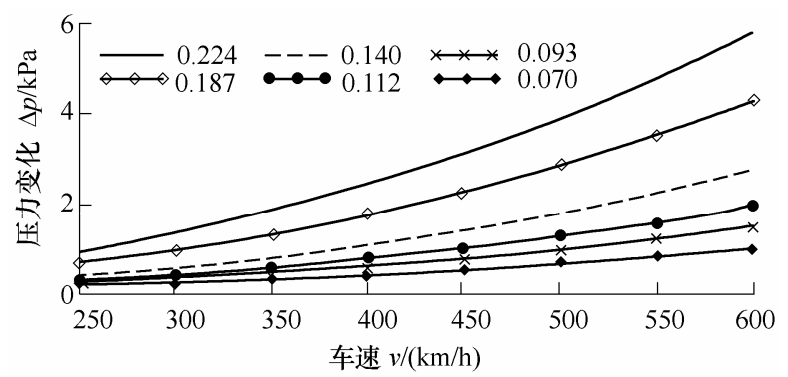

(a) 对最大压力波动的影响

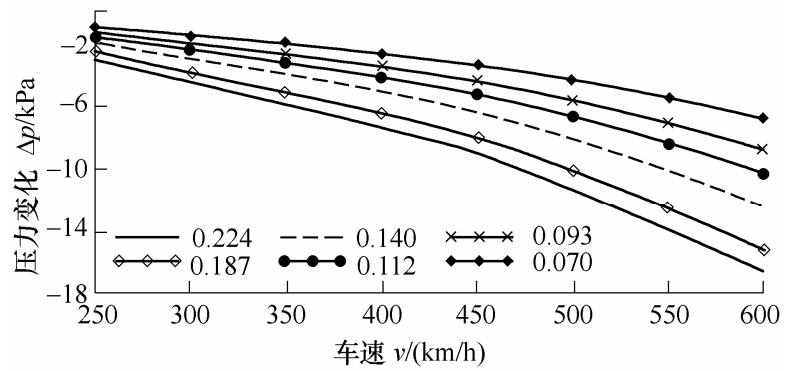

(b) 对最小压力波动的影响

图 5 单车通过隧道时, 第 8 节车厢车外中央处压力 波动峰值与速度的关系

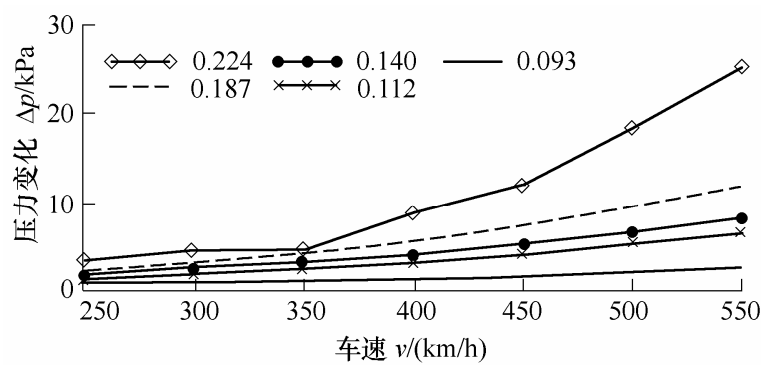

(a) 对最大压力波动的影响

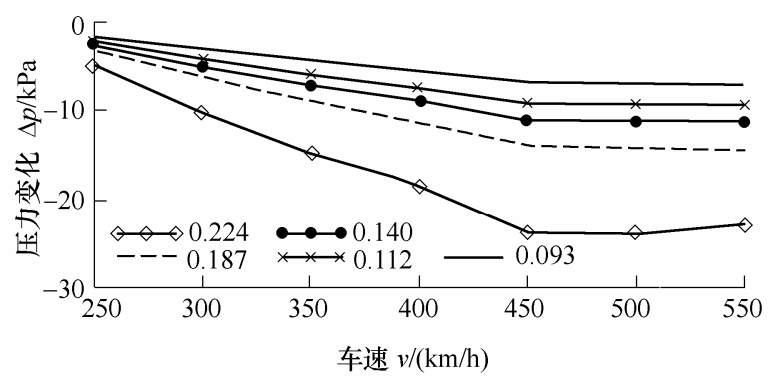

(b) 对最小压力波动的影响

图 6 两列车在隧道内交会时, 第 8 节车厢车外中央处 压力波动峰值与速度的关系

\section{2 阻塞比的影响特性}

图 7 和图 8 表示了阻塞比对车内外压力峰值的 影响特性。可见随阻塞比的增大, 车外的最大压力 波动值增大，而最小压力波动值在减少。仿照上述 压力波动值与列车速度的拟合关系, 可得压力波动 值与阻塞比的依赖关系的幂指数 $n$ 的取值范围。单 列高速列车通过 $2000 \mathrm{~m}$ 隧道过程中, 对于最大压 力波动值 $n=1.257 \sim 1.469$, 对于最小压力波动值 $n=0.896 \sim 0.966$; 而两列车在 $2000 \mathrm{~m}$ 隧道内交会 时, 对于最大压力波动值 $n=1.319 \sim 1.862$, 对于最 小压力波动值 $n=1.030 \sim 1.054$ 。

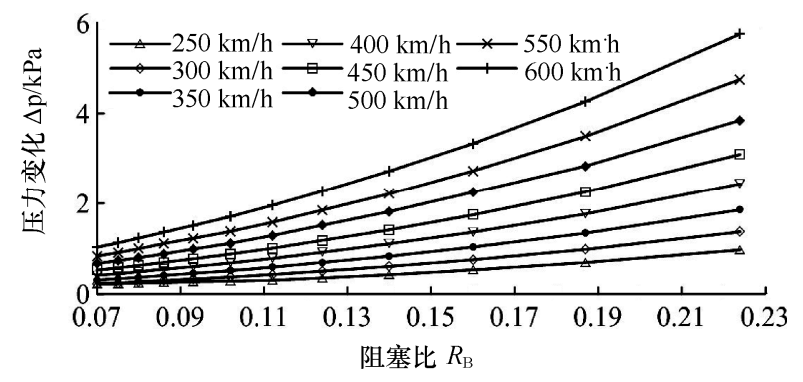

(a) 对最大压力波动的影响

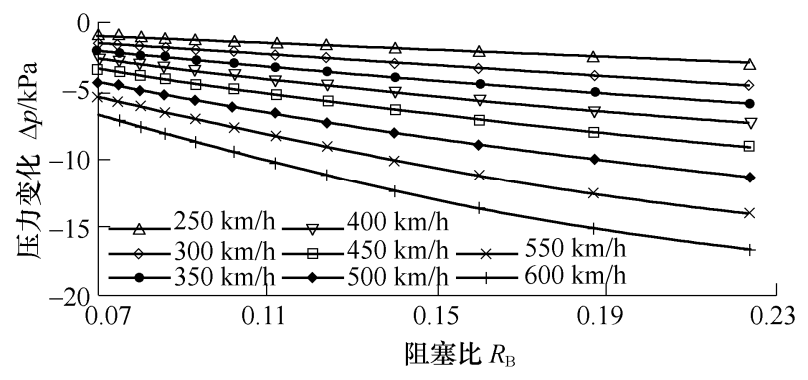

(b) 对最小压力波动的影响

图 7 单车通过隧道时, 第 8 节车厢车外中央处压力 波动峰值与阻塞比的关系 


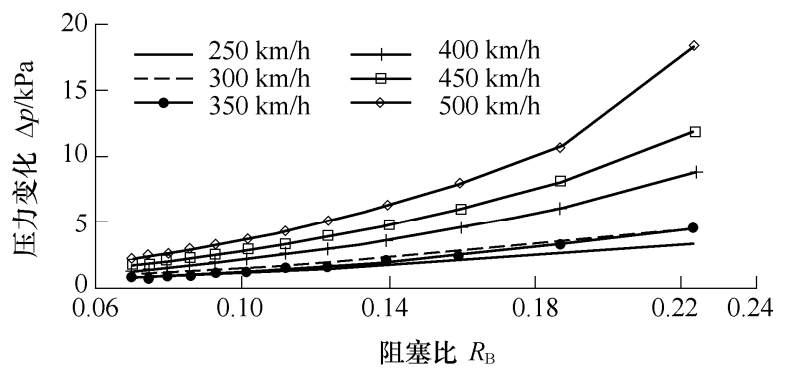

(a) 对最大压力波动的影响

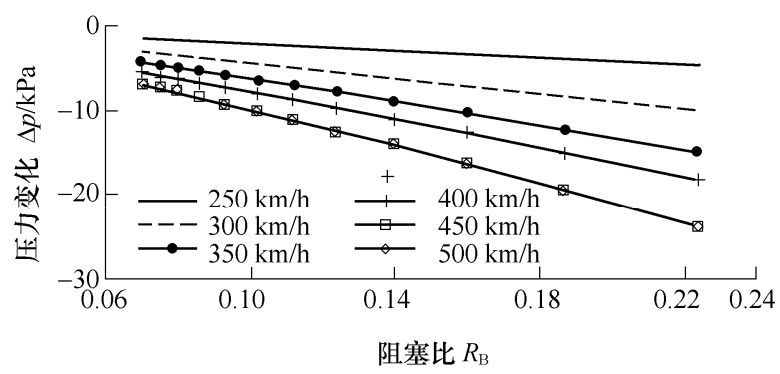

(b) 对最小压力波动的影响

图 8 两列车在隧道内交会时, 第 8 节车厢车外 中央处压力波动峰值与阻塞比的关系

\section{3 单车压力波和会车压力波的比较}

这里, 比较阻塞比 0.112 和不同列车速度条件 下, 单车通过隧道和两列车隧道内交会时的车外压 力波动情况。图 9 所示为不同车厢外中央处压力时

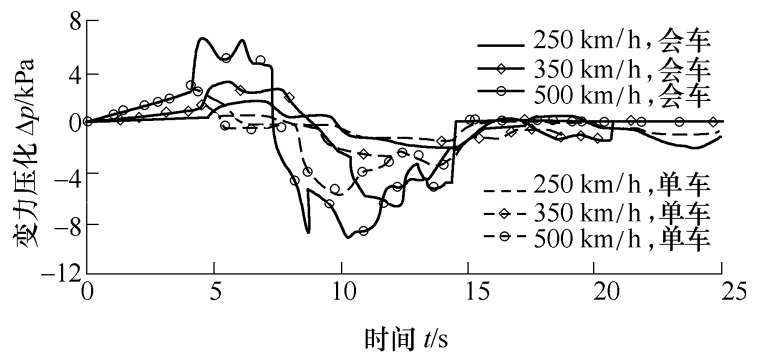

(a) 第 1 节车纵向中央

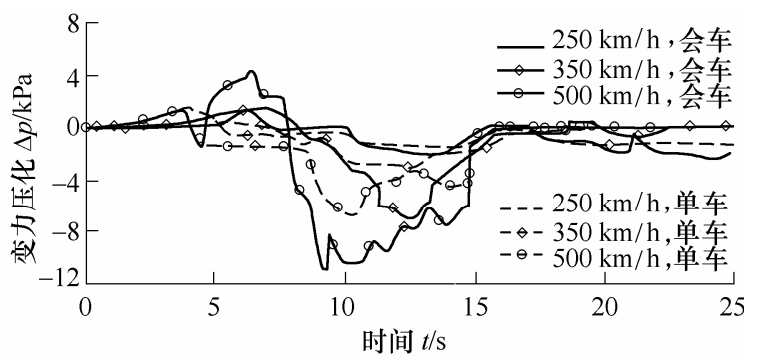

(b) 第 8 节车纵向中央

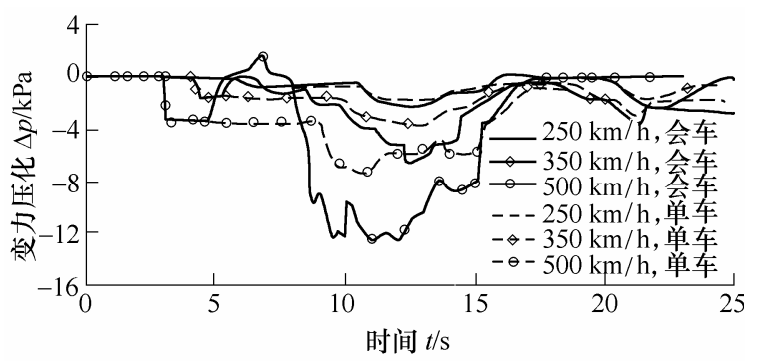

图 9 单车通过及两列车隧道内交会时车外压力 时间历程曲线对比
间历程曲线的对比。图 10 所示为车外最大压力波动 值和最小值压力波动值的对比。从图 9、10 可知, 列车速度对压力时间历程曲线和压力波动峰值的影 响都比较大。由此也可得到类似的结论：隧道会车 压力波比单车压力波更加剧烈, 最大压力波动值和 最小压力波动值比单车过隧道时更大。当列车以 $350 \mathrm{~km} / \mathrm{h}$ 甚至更高速度交会时，车外最小压力波动 值已超过 $-6.0 \mathrm{kPa}$ 。此外, 列车在隧道内运行时, 其车外压力在大多数时间内处于负压状态。且测点位 置距车头距离越远, 处于负压状态的时间越长。尾车 车外压力在列车通过隧道全过程中基本在负压水平。

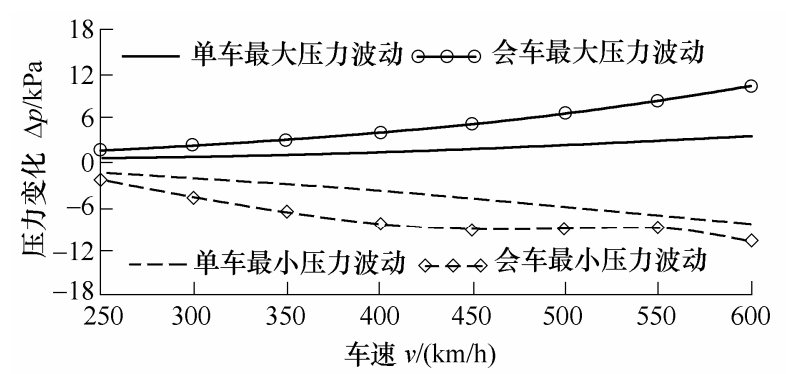

(a) 第 1 节车车外中央处压力波动极值

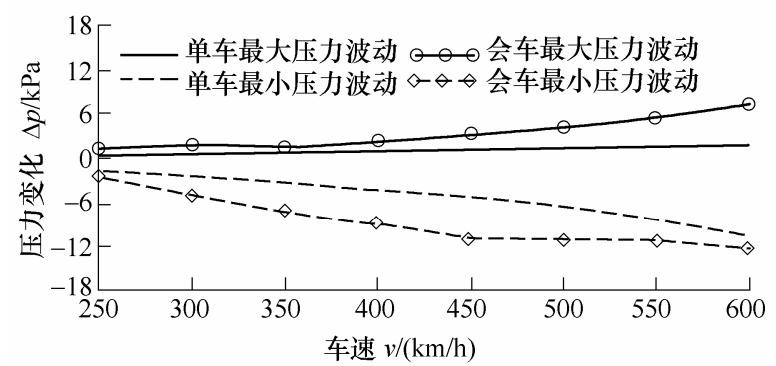

(b) 第 8 节车车外中央处压力波动极值

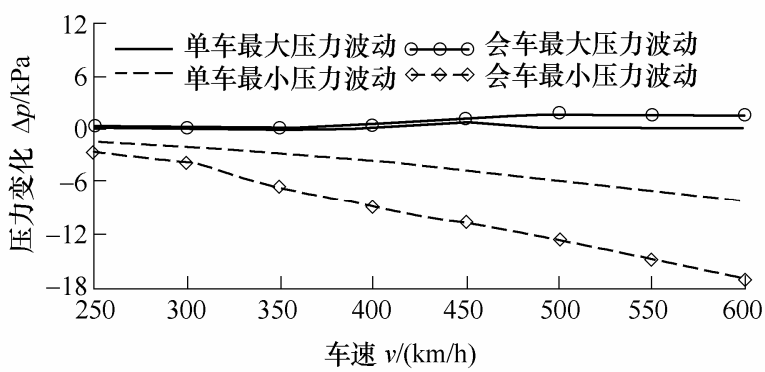

(c) 第 16 节车车外中央处压力波动极值

图 10 单车通过及两列车隧道内交会时车外压力峰值比较

结合以上的参数分析, 在进行高速列车车体及 零部件的设计时, 应该以隧道内交会所产生的压力 波为主要参考依据。

\section{4 结论}

本文首先介绍了基于一维可压缩非定常不等 熵流动模型的广义黎曼变量特征线法计算高速列车 隧道压力波的方法, 并采用日本国铁技术研究所的 
缩尺试验结果和意大利高速铁路线的实车试验结果 验证计算程序的正确性。通过分析全时间区域下隧 道空间中的压力波传播过程, 得出了隧道内会车压 力波比单车压力波剧烈的原因在于同相波的叠加, 即压缩波与压缩波叠加引起压力更剧烈的升高, 而 膨胀波与膨胀波相遇则引起压力更剧烈的降低。此 后本文以中国京沪高速铁路典型隧道为研究对象, 进行了列车速度、阻塞比对车外最大压力和最小压 力波的影响特性研究, 归纳得出相应的影响指数。 结果表明: 对于京沪高铁典型隧道, 单车通过或两 车在隧道内交会时, 其压力波动峰值近似与车速的 平方成正比。而压力波正峰值近似与阻塞比的 1.3 1.8 次方成正比, 压力波峰值与阻塞比近似成正比关 系。通过对比不同列车速度下单车过隧道和隧道交 会两种情形的压力波变化特性, 发现会车压力波动 显著高于单车, 后续工作应结合实际运营情况以会 车工况为主进行研究。建议组织开展超高速列车实 车或模型试验, 探明高速列车隧道压力波典型参数 在超高速条件下的变化特征, 为我国高速铁路进一 步提速理论及数值模拟提供依据。

\section{参 考 文 献}

[1] 肖京平, 黄志祥, 陈立. 高速列车空气动力学研究技术 综述 $[\mathrm{J}]$. 力学与实践, 2013, 35(2): 1-12.

XIAO Jingping, HUANG Zhixiang, CHEN Li. Review of aerodynamic investigations for high speed[J]. Mechanics in Engineering, 2013，35(2): 1-12.

[2] 梅元贵, 周朝晖, 许建林. 高速铁路隧道空气动力 学[M]. 北京: 科学出版社, 2009 .

MEI Yuangui, ZHOU Chaohui, XU Jianlin. Aerodynamics of high-speed railway tunnel[M]. Beijing: Science Press, 2009.

[3] AHMED S R, GAWTHORPE R G, MACKRODT P A. Aerodynamics of road-and rail vehicles[J]. Vehicle System Dynamics, 1985, 14: 319-392.

[4] YAMAMOTO A. Pressure variations aerodynamic drag of trains and natural ventilation in SHINKSEN type tunnel[J]. JNR, 1974(4): 207-214.

[5] IIDA M, MAEDA T. Numerical calculation of pressure transients in railway tunnels[J]. RTRI Report, 1990, 4(7): 54-62.

[6] SCHULTZ M, SOCKEL H. Pressure transients in short tunnels $[\mathrm{C}] / /$ The 7 th International Symposium on Aerodynamics and Ventilation of Vehicle Tunnels. 1991,

Elsevier Science Publishers Ltd., London, 1991: 221-238.

[7] VARDY A E. The use of airshafts for the alleviation of pressure transients in tunnels[C]//Proceeding 2nd
ISAVVT, March 1976, BHRA Fluid Engineering, Cambridge, Paper C4, 1976: 55-69.

[8] HENSON D A, GAWTHORPE R G, POPE C W, et al. Aerodynamics and ventilation of a proposed channel tunnel[C]//The 4th ISAVVT, BHRA Fluid Engineering,

March, 1982, Cranfield, York, 1982: 23-25.

[9] WOODS W A, POPE C W. A generalized flow prediction method for the unsteady flow generated by a train in a single tunnel[J]. Journal of Wind Engineering and Industrial Aerodynamics, 1981, 7(3): 331-360.

[10] SANETOSHI S, MASANOBU I, HIROSHI K. Numerical simulation of 1-D unsteady compressible flow in railway tunnels $[\mathrm{J}]$. Journal of Environment and Engineering, 2011, 6(4): 723-738.

[11] WOODS W A. On the range of validity of simplified one dimensional theories for calculating unsteady flow in railway tunnels[C]// The 3rd International Symposium on the Aerodynamics and Ventilation of Vehicle Tunnels, 1979, Cranfield Bedford England: BHRA, Paper D2, 1979: $115-150$.

[12] WOODS W A, POPE C W. A generalized flow prediction method for the unsteady flow generated by a train in a single tunnel[J]. Journal of Wind Engineering and Industrial Aerodynamics, 1981, 7: 331-360.

[13] STEINRÜCK P, SOCKEL H. Further calculations on transient pressure alleviation and simplified for initial tunnel design[C]// The 5th International Symposium on the Aerodynamics and Ventilation of Vehicle Tunnels, 1985, Cranfield Bedford England: BHRA, Paper E4, 1985: 317-342.

[14] 梅元贵. 高速铁路隧道压力波数值模拟研究[D]. 成都: 西南交通大学, 1997.

MEI Yuangui. A study on numerical simulation of high-speed trains induced pressure waves in a single bore tunnel[D]. Chengdu: Southwest Jiaotong University, 1997.

[15] 陶泽平, 杨志刚, 陈羽. 列车隧道交会最不利长度数值 模拟研究[J]. 佳木斯大学学报, 2013, 31(1): 7-11.

TAO Zeping, YANG Zhigang, CHEN Yu. Numerical simulation on the most unfavorable tunnel length of high speed trains passing by each other in tunnel[J]. Journal of Jiamusi University, 2013, 31(1): 7-11.

[16] 王一伟, 杨国伟, 黄晨光, 等. 隧道长度对高速列车交 会压力波的影响研究 [J]. 中国科学: 技术科学, 2012, 42(1): 82-90.

WANG Yiwei, YANG Guowei, HUANG Chenguang, et al. Influence of tunnel length on the pressure wave generated by high-speed trains passing each other[J]. 
Science China Technology Science, 2012, 42(1): 82-90.

[17] KRAJNOVIC S, HEMIDA H, DIEDRICHS B. Time-dependent simulations for the directional stability of high speed trains under the influence of cross winds or cruising inside tunnels[C/CD $] / /$ Fluid Dynamics Applications in Ground Transportation, October 26-27, 2005, Lyon, France.

[18] KWON H B, KIM T K, LEE D H, et al. Numerical simulation of unsteady compressible flows induced by a high-speed train passing through a tunnel[J]. Proceedings of the Institution of Mechanical Engineers, Part F: Journal of Rail and Rapid Transit, 2003， 217(2): 111-124.

[19] TAKANOBU O, FUJII K. Numerical investigation of three-dimensional compressible flows induced by a train moving into a tunnel[J]. Computers \& Fluids, 1997, 26(6): 565-585.

[20] MOK J K, YOO J. Numerical study on high speed train and tunnel hood interaction[J]. Journal of Wind Engineering and Industrial Aerodynamics，2001， 89(1): 17-29.

[21] 施成华, 杨伟超, 彭立敏, 等. 高速铁路隧道空气动力 效应对水沟盖板稳定性的影响研究 $[\mathrm{J}]$. 铁道学报, 2012，34(1): 103-108.

SHI Chenghua, YANG Weichao, PENG Limin, et al. Study on aerodynamci influenceon on stability of ditch coversin high-speed railway tunnels[J]. Journal of the China Railway Society, 2012，34(1): 103-108.

[22] 李人宪, 赵晶, 刘杰, 等. 高速列车会车压力波对侧窗 的影响 $[\mathrm{J}]$. 机械工程学报, 2010, 46(4): 87-92.

LI Renxian, ZHAO Jing, LIU Jie, et al. Influence of air pressure pulse on side windows of high-speed trains passing each other[J]. Journal of Mechanical Engineering, 2010, 46(4): 87-92.

[23] 费瑞振, 彭立敏, 杨伟超, 等. 高速铁路隧道空气动力 效应对人员安全的影响研究 $[\mathrm{J}]$. 中国安全科学学报, 2013，23(7): 79-84.

FEI Ruizhen, PENG Limin, YANG Weichao, et al. Study on aerodynamic force effect on personnel safety in high-speed railway tunnel[J]. China Safety Science Journal，2013， 23(7): 79-84.

[24] 李人宪, 关永久. 高速列车隧道会车压力波动问题 $[\mathrm{J}]$. 机械工程学报, 2012, 48(20): 127-134.

LI Renxian, GUAN Yongjiu. Investigation of air pressure pulse when two high-speed trains passing by each other in tunnel[J]. Journal of Mechanical Engineering, 2012, 48(20): $127-134$.

[25] SAITO S, IIDA M. Development and verification of numerical simulation of pressure changes in underground high-speed railways[J]. RTRI Report, 2006, 20(1): 29-34.

[26] WILLIAM-LOUIS M J P , GRÉGOIRE R. 1-d calculations of pressure fluctuation outside and inside a pressure sealed high-speed trainset traveling through tunnels[C]/Notes on Numerical Fluid Mechanics and Multidisciplinary Design 79, Berlin: Springer-Verlag, 2002, 342-357.

[27] VARDY A E, ANANDARAJAH A. Initial design consideration for rail tunnel aerodynamics and thermodymaics[C]// The 4th International Symposium on the Aerodynamics and Ventilation of Vehicle Tunnels. Cranfield Bedford England: BHRA, 1982. Paper H2, 1982: 353-365.

[28] POPE C W, GAWTHORPE R G, RICHARDS. An experimental investigation into the effect of train shape on the unsteady flows generated in tunnels[C]// The 4th International Symposium on the Aerodynamics and Ventilation of Vehicle Tunnels. Cranfield Bedford England: BHRA, 1982. Paper C2, 1982: 107-128.

作者简介: 贾永兴, 男, 1982 年出生, 博士研究生。主要研究方向为高 速列车空气动力学。

E-mail: jiayongxing2004@163.com

杨永刚, 男, 1990 年出生。主要研究方向为高速列车空气动力学。

E-mail: 993979024@qq.com

梅元贵(通信作者), 男, 1964 年出生, 教授, 博士研究生导师。主要研 究方向为高速列车空气动力学。

E-mail: meiyuangui@163.com 